\title{
La cession de créances au médecin
}

\author{
Dr PHILIPPE GEISSBÜHLER, Dr GRÉGOIRE GEISSBÜHLER \\ Rev Med Suisse 2018; 14: 1048-9
}

\section{INTRODUCTION}

La cession de créances: voilà un sujet rébarbatif qui ne concerne que le médecin installé facturant ses honoraires, jamais traité pendant les études ou la vie «intrahospitalière», où son abord serait de toute façon prématuré. Il y est question de la LAMal, du tiers payant, du tiers garant, des relations aux assurances, de remboursement de factures, d'impayés et de contentieux, toutes choses éloignées de nos préoccupations cliniques habituelles.

Lorsqu'on facture, le tiers garant est la règle. Le tiers, c'est l'assurance. Le patient paie son médecin et l'assurance rembourse le patient. Le problème surgit lorsque le patient ne paie pas, ou préfère utiliser l'argent de l'assurance à d'autres fins. Dans ces cas, le médecin aimerait bien que l'assurance le paie directement. Mais on ne peut pas passer simplement au tiers payant.

Depuis quelques années, il existe une lutte pour l'obtention du détail des données de facturation, qui permettent de négocier la valeur du point Tarmed. Les assurances proposent aux médecins intéressés de leur transmettre ces données en échange de la possibilité de facturer en tiers payant. A ceux qui ne souhaitent pas s'engager dans cette voie, il reste la cession de créances.

Or, dans ce domaine méconnu des médecins, on voit quelquefois des prises de position étranges des assurances. Ainsi Helsana qui en 2015 envoie une circulaire par courriel en enjoignant les médecins à refaire tous les six mois les cessions de créances, ou EGK qui exige pour une patiente l'envoi d'une cession originale avec chaque facture d'honoraires.

Ceci nous donne l'occasion de faire cet article de mise au point; un travail interprofessionnel, interfacultaire et intra-familial.

\section{BASES LÉGALES}

L'art. 42 al. 1 LAMal permet au patient de céder au médecin la créance qu'il détient contre son assureur. On applique alors les règles de la cession de créances au sens des articles 164 à 174 du Code des obligations (CO)..$^{1}$ En particulier, la «dation en vue d'encaissement» de l'art. $172 \mathrm{CO}^{2}$ permet au médecin de réclamer directement à l'assureur le paiement de la prestation, à concurrence de ce que le patient pourrait obtenir. Le paiement éteint simultanément la dette de l'assureur envers le patient et la dette du patient envers le médecin. ${ }^{3}$

\section{PRINCIPES}

Les règles essentielles sont les suivantes:

1. Accord du patient: Il n'est pas possible que le patient cède sa créance sans y avoir consenti.

2. Contenu: Seules les créances résultant des soins fournis par le médecin lui-même sont concernées. Le patient ne peut céder les créances d'assurance-maladie ayant une autre source: par exemple celles découlant des soins procurés par un autre médecin. ${ }^{4}$

3. Moment et durée: La cession peut concerner tant les créances déjà existantes que les créances futures. Une cession future illimitée n'est pas possible, mais une cession pour cinq à dix ans reste admissible. $^{5}$

4. Forme écrite: Toute cession de créances, qu'elle soit dans un cadre médical ou non, est soumise à la forme écrite, c'est-à-dire qu'elle doit être faite sur un support papier (même dactylographié), et impérativement signée à la main par le patient (et/ou son représentant légal: parent, curateur, etc.). La signature du médecin n'est pas requise mais peut être utile. ${ }^{6}$

5. Notification: Enfin, la cession de créances doit être notifiée à l'assureur. La notification peut être faite par le patient ou le médecin lui-même. ${ }^{7}$ Nous conseillons au médecin d'y procéder lui-même. La notification doit être renouvelée à chaque changement d'assureur.

La formulation est libre, dès lors qu'elle exprime clairement la volonté de procéder à la cession et se conforme aux critères que nous avons exposés. Par exemple:
En application de l'art. 42 al. 1 LAMal, je, soussigné(e), [Patient], cède à [Médecin] toutes les créances présentes et futures contre mon assureur maladie découlant des prestations fournies par [lui/elle], en vue du paiement de celles-ci. Cette cession globale prendra fin au plus tard le [date]. [Médecin] restera titulaire des créances cédées jusqu’à cette date.

[Médecin] est autorisé(e) à notifier la présente cession à tout assureur maladie concerné.

Fait à [lieu], le [date]

[Signature du Patient]

[Signature du Médecin]

Quelques éléments supplémentaires méritent d'être relevés.

L'assureur n'a pas son mot à dire dans la cession, qui peut être faite même sans son consentement. L'assureur ne peut notamment pas restreindre unilatéralement la possibilité ou les modalités de cession par exemple en fixant une durée ou un montant maximum. ${ }^{8}$

En cas de remboursement par l'assureur au patient directement, après la notification, cela ne suffit pas à le libérer. Le médecin est donc toujours fondé à réclamer le paiement directement à l'assureur. Celui-ci se retournera ensuite contre le patient, sans que le médecin ne soit concerné. ${ }^{9}$

Si l'assureur a le droit de refuser le paiement au patient de tout ou partie des prestations reçues - par exemple la franchise et la quote-part à sa charge, ou les frais pour des prestations non couvertes par la LAMal - ce refus est également opposable au médecin..$^{10}$ Le non-paiement des primes n'est toutefois pas un motif valable de refus. ${ }^{1}$

Le patient et le médecin ont tous deux le droit de résilier, en tout temps et sans motifs, le mandat qui les lie. Si cela devait arriver, les créances antérieures à la résiliation, pour les soins déjà prodigués, ne sont pas affectées. Le médecin conserve donc le droit d'en réclamer le paiement à l'assureur. 
La cession de créances offre ainsi au médecin un moyen pratique et peu complexe de se prémunir contre le risque financier représenté par les patients insolvables ou peu enclins à s'acquitter de leurs dettes.

1 ATF 135 V 2, consid. 6.1.

2 Figure par laquelle un débiteur cède à son créancie une créance détenue contre un tiers, le montant obtenu servant ensuite à éteindre la dette initiale (ATF 131 III 217, consid. 4.2).
3 Probst Thomas, in Thévenoz Luc / Werro Franz (édit.) Commentaire romand, $\mathrm{CO} I$ (Code des obligations, art. 1-529 CO), 2e éd., Bâle, 2012 (cité: CR CO I-AUTEUR), CO 172 N 1 ss; ATF 135 V 2, consid. 6.1.

4 Geissbühler Grégoire, Tiers garant, tiers payant (42 LAMal) - Un point de vue de droit des obligations, PJA 2015, p. 1014.

5 Geissbühler (n. 5), p. 1015; CR CO I-Probst (n. 4), CO $164 \mathrm{~N} 42$.

6 Art. 165 CO; CR CO I-Probst (n. 4), CO 165 N 2; ATF 122 III 361, consid. 4.

7 Art. 167 CO; CR CO I-Probst (n. 4), CO 167 N 5

8 Geissbühler (n. 5), p. 1015.

9 CR CO I-Probst (n. 4), CO 167 N 2 ss.

10 Art. 169 CO.

11 Art. 64a LAMal; FF 20095975.

\section{DR PHILIPPE GEISSBÜHLER}

Spécialiste médecine interne 25 place de la Gare

2800 Delémont

geissbuhler.ph@hin.ch

\section{DR IUR. GRÉGOIRE GEISSBÜHLER}

Avocat-stagiaire (LALIVE, Genève)

chargé de cours à l'Université de Lausanne gregoire.geissbuhler@unil.ch 